\title{
Hybrid Medical Image Segmentation based on Fuzzy Global Minimization by Active Contour Model
}

\author{
J. Umamaheswari \\ Research Scholar, Department of Computer \\ Science, Dr. G.R.D College of Science, \\ Coimbatore, Tamilnadu, India
}

\author{
G. Radhamani \\ Director, Department of Computer Science \\ Dr. G.R.D College of Science, \\ Coimbatore, Tamilnadu, India
}

\begin{abstract}
This paper provides new hybrid medical image segmentation based on Global Minimization by Active Contour (GMAC) method and Spatial Fuzzy C Means Clustering method (SFCM) tailored to CT imaging applications. GMAC is the unification of image segmentation and image denoising, which is a combination of snake, Rudin-Osher denoising and the Mumford Shah model. Here globalization of contour is applied which normalizes the threshold to form a cluster by spatial fuzzy means. By allowing the Active contour to detect the region of features that is to be segmented is spatial functioned by fuzzy $\mathrm{c}$ means is applied for fining the segmentation results. Our method is compared with other methods like Adaptive Threshold (AT), Edge detecting, Region Growing by Adaptive (RGA) Threshold to prove the efficiency. We validate the new approach with the parameters in terms of energy level, Relative Entropy (RE), Discrete Entropy(DE), Mutual information(MI), Evaluation time(ET). The experimental result shows that the proposed model works efficiently.
\end{abstract}

\section{Keywords}

Fusion technique, Global Minimization by active contour, spatial fuzzy clustering, Adaptive thresholding, Medical image.

\section{INTRODUCTION}

Segmentation is widely used in medical imaging modalities such as PET, CT, MRI, etc. The segmentation plays a main role in medical applications such as surgical planning, abnormality detection and treatment progress monitoring. The image captured is classified by clustering and segmenting. The clustering consists of white matter, grey matter and background objects. In such cases segmentation is processed by using contour methods to segment the images for fine quality image.

Though multiphase Mumford shah's (MS) [3],[4],[5] model has multicontour which overlaps the points that occur and minimize the energy value by weighted total variation value [1],[10] which is a disadvantage for medical imaging . In this paper GMAC combined with SFCM is proposed. hybrid method that combines region-based fuzzy clustering method called Enhanced Possibility Fuzzy C Means (EPFCM) and Generalized Gradient vector flow (GGVF) snake model for segmenting tumor region on MRI images effectively. Region based fuzzy clustering is used for initial segmentation of tumor then result of this is used to provide initial contour for GGVF snake model, which then determines the final contour for exact tumor boundary for final segmentation [14]. A new hybrid medical image segmentation method in the level-set framework is proposed. The method uses both the object boundary and region information to achieve robust and accurate segmentation results [15].
In our proposed method the contour is applied globally to the image as seedpoint growing in which the edge is detected, then the clusters are applied to the object by threshold values, This spatially grouped value are contour to give a fine segmented images.

This paper is organized as follows, Section 2 describes the hybrid model GMAC, Section 3 describes the SFCM model. Section 4 defines the criteria for quantifying the performance of the proposed model and presents experimental results for CT (Computer tomography) images Section 5. Concludes the paper.

\section{GMAC}

In active contour model, the segmented images play a very important role. Here, different initial conditions evolution will give different segmented region. The accured results will not be satisfactory. This unsatisfactory result is the minimization problem of the active contour. Therefore energy minimization could be trapped into a local minimum by snake model.

A few algorithms were proposed in $[5,1]$ to determine the global minimization which is to modify the ROF (Region of Features) energy given below,

$$
E_{R O F}(v, \lambda)=\int_{\Omega}|\nabla v|+\frac{\lambda}{2} \int_{\Omega}|v-f|^{2}
$$

The variation (L1) is replaced by weighted variation (L2), changing the measure in terms of fidelity (f) from the square of $L_{2}$ to the $L_{1}$.

$$
E_{2}(v, \lambda)=\int_{\Omega}^{c} g(f)|\nabla v|+\lambda \int_{\Omega}^{c}|v-f|^{2}
$$

Where, $g(f)=1 /\left(1+\beta|\nabla f|^{2}\right)$

If $\breve{v}$ is the characteristic function of a set with boundary given by the curve $C$ the energy level $E_{2}$ is the same as the energy level of the active contour occurred

$$
E_{\Lambda C}=\int_{c} g(f) d s
$$

with $f$ approximated $\left(L_{l}\right)$ by a binary function of a region $\Omega C$. Numerically, the minimization is a convex problem. So the unification of images which is to be enhanced(3), for smoothing the contour level in given images the Gaussian filter is applied .The global minimization active contour 
avoids the uncertainty disturbances, So FCM with spatial initialization is applied as clusters.

\section{SFCM}

FCM is a method of clustering starts with the center point of the cluster. These cluster point will not be accurate, so FCM assigns membership rank for every cluster at every point. FCM iteratively moves the cluster to the correct place within a dataset. This iteration is based on minimizing an objective function that symbolizes the distance from any given data point [6,7]. To normalized the datapoint occurred in the images a gradient function using FCM is clustered, when pixels are close to the centroid of pixels assigned as 0,1 which optimize the function as follows:

$$
J=\sum_{p=1}^{M} \sum_{q=1}^{N} u_{p q}^{o}\left\|y_{p}-x_{q}\right\|^{2}
$$

where ${ }_{U p q}$ represents the membership of pixel $y_{p}$ in the $p^{\text {th }}$ cluster, $v_{i}$ is the $q^{\text {th }}$ cluster center, $\|\ldots\|$ is a norm metric, and ' 0 ' is a constant. The function is minimized when pixels are close to the centroid of their clusters, that are assigned by membership values $(0,1)$. Low membership values are assigned to the pixels with data far from the centroid. The probability represents the membership function is a pixel that belongs to a specific cluster. This probability is dependent solely on the distance between the pixel and each individual cluster dependent according the image.

$$
\begin{aligned}
& u_{p q}=\frac{1}{\sum_{r=1}^{o}\left(\frac{y_{q}-x_{p}}{y_{q}-x_{r}}\right)^{2 / o-1}} \\
& x_{p}=\frac{\sum_{q=1}^{M} u_{p q}^{o} y_{p}}{\sum_{q=1}^{M} u_{p q}^{o}}
\end{aligned}
$$

Starting with an point for each cluster, the FCM converges to endpoint $v_{i}$, representing the cost function.

When the images are highly interrelated with the neighboring pixels, that shows the similar feature values which belong to the same group a spatial function [3] is defined as follows:

$$
g_{i j}=\sum_{L \in A B\left(x_{j}\right)} M_{i k}
$$

where $\mathrm{AB}\left(\mathrm{x}_{\mathrm{j}}\right)$ represents a square window centered on pixel $\mathrm{x}$ in the spatial domain. The spatial function gij represents the probability and pi belongs to the cluster. The spatial function of a pixel for a cluster is large if the majority of its neighborhood belongs to the same cluster [13]. The spatial function is incorporated into membership function as follows:
$M_{i j}=\frac{g_{i j}^{p} M_{i j}^{q}}{\sum_{k=1}^{c} g_{k j}^{p} M_{k j}^{q}}$

where $\mathrm{p}$ and $\mathrm{q}$ are parameters to control the functions that strengthen the membership while the clustering results remain unchanged.

The clustering is a two-stage process at each iteration.

1. Spectral domain is calculated using membership function.

2. The membership information of each pixel is mapped to the spatial domain.

The FCM iteration proceeds with the new membership that is incorporated with the spatial function. When the maximum difference between two cluster centers at two successive iterations is less than a threshold, the iteration is stopped. After the convergence, defuzzification is applied to assign each pixel to a specific cluster for which the membership is maximal.

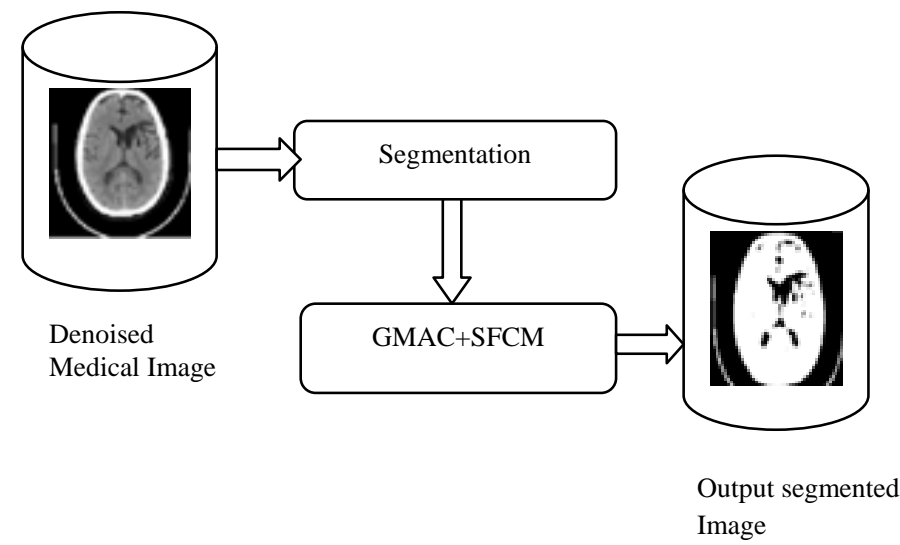

Fig.1 Proposed Hybrid medical image segmentation model

The Figure 1 shows the hybrid medical image segmentation model. The idea is to minimizing the energy which gives a quality image that occur . The optimization property of FCM is improved when it is combined with spatial information (SFCM).

\section{EXPERIMENTAL RESULTS}

The performance of our proposed model is evaluated by medical brain images compared with other methods such as AT(Adaptive Threshold),Edge Detecting, RGA(Region Growing Adaptive Threshold) AT+FL (Adaptive Threshold + Fuzzy levelset), RGA+FL, The parameters used to prove the proposed method is efficient by Energy, Relative Entropy(RE), Mutual Information(MI) and Elapsed time(ET). Experiments are conducted using matlab 7.1. Benchmark images taken for analysis are DICOM medical images. To test the accuracy of the segmentation algorithms:

i) A denoised medical image is taken as input.

ii) The proposed hybrid model is applied to the medical image.

iii) The performance evaluation is obtained by the statistical method like Energy, Relative Entropy (RE), Mutual 
Information (MI) and Elapsed Time (ET) which is low when compared with other methods.

The reconstruction of an image has the dimensions of 256 pixel intensity. The medical images in this contain a wide variety of subject matters and textures. Most of the images used are brain images with defect and without defected images. The Energy value must be low for an medical image, Relative Entropy (RE), Discrete Entropy (DE), Mutual Information (MI) and Elapsed Time (ET).must be less value for a better segmentation algorithm.

\section{a) Energy}

The gray level energy indicates how the gray levels are distributed. It is formulated as,

$$
E(x)=\sum_{i=1}^{x} p(x)
$$

Where $\mathrm{E}(\mathrm{x})$ represents the gray level energy with 256 pixels and $\mathrm{p}$ (i) refers to the probability distribution functions, which contains the histogram counts. The energy reaches its maximum value of 1 when an image has a constant gray level.

The larger energy value corresponds to the lower number of gray levels, which means the defect is simple and cured by medicine or with simple medical applications. The smaller energy value corresponds to the higher number of gray levels, which means the defect is complex cured only by surgery.

Table. 1 Energy value for different segmentation algorithm

\begin{tabular}{|c|c|c|c|c|c|c|}
\hline & $\begin{array}{l}\text { AT } \\
(\mathbf{E}-01)\end{array}$ & $\begin{array}{l}\text { Edge } \\
\text { (E-01) }\end{array}$ & $\begin{array}{l}\text { RGA } \\
\text { (E-01) }\end{array}$ & $\begin{array}{l}\mathbf{A T}+\mathbf{F L} \\
(\mathbf{E}-01)\end{array}$ & $\begin{array}{l}\text { RGA+FL } \\
\text { (E-00) }\end{array}$ & $\begin{array}{l}\text { gmac+sfcm } \\
\text { (E-01) }\end{array}$ \\
\hline $\mathbf{E}$ & 6.67 & 8.73 & 6.48 & 6.64 & 0.602 & 4.40 \\
\hline RE & 5.70 & 2.49 & 5.79 & 8.22 & 0.702 & 9.80 \\
\hline DE & 7.58 & 3.59 & 8.36 & 1.6 & 1.21 & 2.28 \\
\hline MI & 1.76 & 5.87 & 2.76 & 6.17 & $1.66 \mathrm{E}$ & 1.50 \\
\hline ET & 4.58 & 4.88 & 6.89 & 8.61 & 8.89 & 5.7 \\
\hline
\end{tabular}

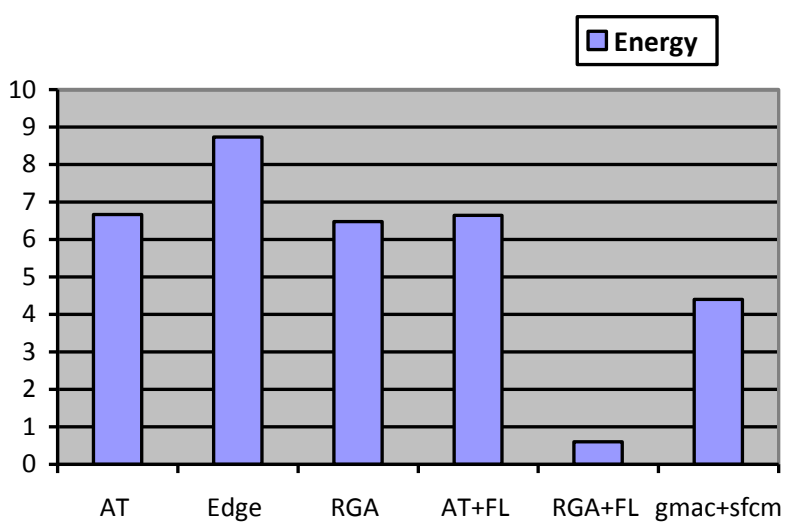

Fig. 2 -Energy value for proposed segmentation algorithm

The figure 2 and table 1 shows the energy value is efficient for the proposed hybrid method.

\section{b) Relative Entropy (RE)}

Two discrete probability distributions of the images have the probability functions of $p$ and $q$, the relative entropy of $p$ with respect to $\mathrm{q}$ is defined as the summation of all possible utcomes that occur. This entropy gives the features which are similar or any nearer value to the common features of the images with are related (10)

$$
d=\sum_{i=1}^{k} p(i) \log _{2} \frac{p(i)}{q(i)}
$$

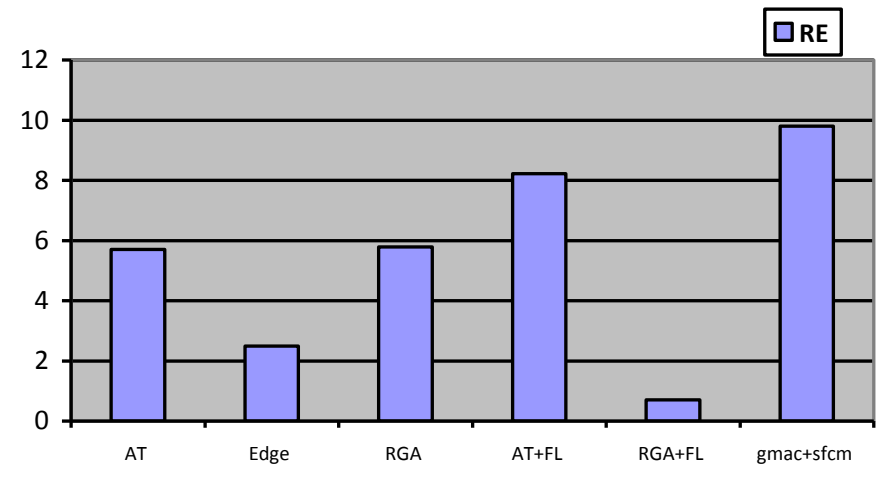

Fig. 3 Relative Entropy value for proposed segmentation algorithm

The figure 3 and table 1 shows the RE value for proposed hybrid segmentation model gives suitable results.

\section{c) Discrete Entropy (DE)}

The discrete entropy is the measure of image information content, which is interpreted as the average uncertainty of information source. It is calculated as the summation of the products of the probability of outcome multiplied by the log of the inverse of the outcome probability, taking into consideration all possible outcomes $\{1,2, \ldots, \mathrm{n}\}$ in the event $\left\{\mathrm{x}_{1}, \mathrm{x}_{2}, \ldots, \mathrm{x}_{\mathrm{n}}\right\}$, where $\mathrm{n}$ is the gray level; $\mathrm{p}(\mathrm{i})$ is the probability distribution, considering all histogram counts. The discrete entropy is a measure to know how many bits are needed for coding the image data, which is a statistical measure of randomness. The maximal entropy occurs when all potential outcomes are equal. When the outcome is certainty, the minimal entropy occurs which is equal to zero. The discrete entropy represents average amount of information conveyed from each individual image (11),

$$
H(x)=\sum_{i=1}^{k} p(i) \log _{2} \frac{1}{p(i)}=-\sum_{i=1}^{k} p(i) \log _{2} p(i)
$$

\section{d.) Mutual Information (MI)}

The notion of the mutual information can be applied as another objective metric. The mutual information acts as a symmetric function (12),

The following figure 4 and table 1 shows the DE value for proposed hybrid model gives suitable results. 


$$
\begin{aligned}
& I(X, Y)=\sum_{X Y} P_{X Y}(X, Y) \log _{2} \frac{P_{x y}(X, Y)}{P_{x}(X) P_{y}(Y)} \\
& =-\sum_{x} P_{x}(X) \log _{2} P(X)+\sum_{x \cdot y} P_{x y}(X, Y) \log _{2} \frac{P_{x y}(X, Y)}{\left.P_{x}(X) P_{y} Y\right)} \\
& =H(X,)-H(X \mid Y)
\end{aligned}
$$

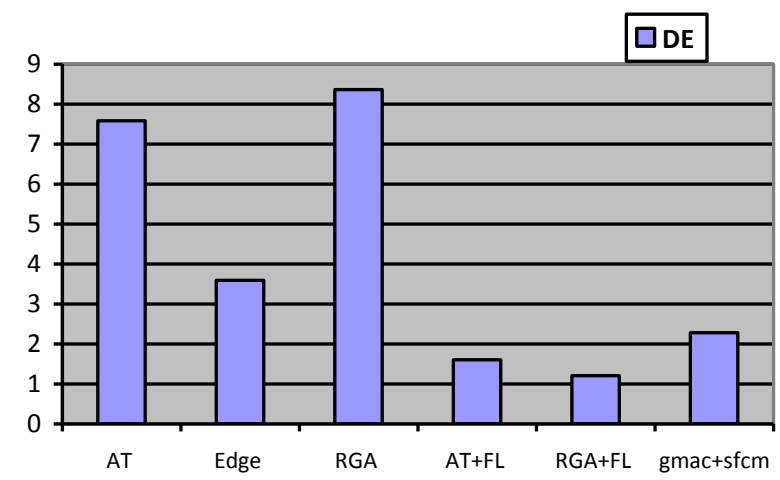

Fig. 4 Discrete Entropy value for proposed segmentation algorithm

Where $\mathrm{I}(\mathrm{X}, \mathrm{Y})$ represents the mutual information, $\mathrm{H}(\mathrm{X})$ and $\mathrm{H}(\mathrm{X} \mid \mathrm{Y})$ are entropy and conditional entropy values. It is interpreted as the information that $\mathrm{Y}$ can tell about $\mathrm{X}$ and the measure of reduction in uncertainty of $\mathrm{X}$ due to the existence of Y. At the same time, it also shows the relationship of the joint and product distributions.

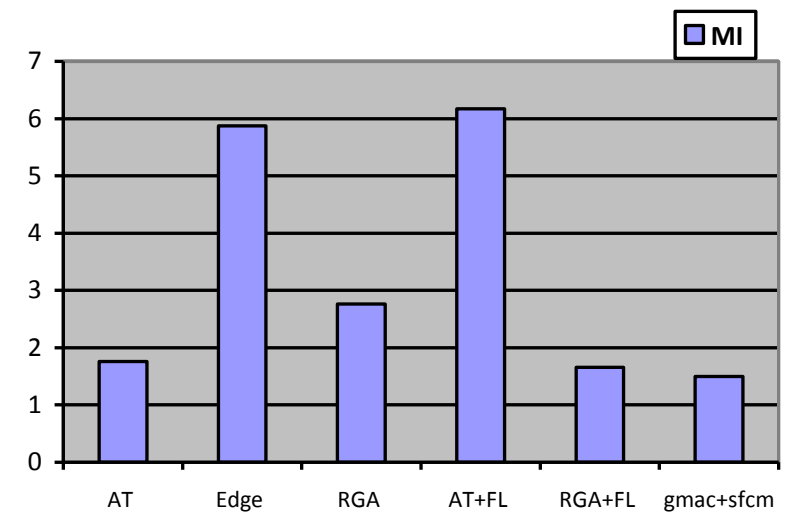

Fig. 5 Mutual Information value for proposed segmentation algorithm

The following figure 5 and table1 shows the MI value for proposed hybrid model gives suitable results.

\section{e.) Evaluation Time}

Evaluation Time (ET) of a filter is defined as the time taken by a digital computing platform to execute the filtering algorithms when no other software, except the operating system (OS), runs on it. Though ET is not only dependant on the clock time. Rather, in addition to the clock-period, it depends on the memory-size, the input data size, and the memory access time. However, the measure ET is very important in case of real-time application.

The execution time taken by a filter should be low for online and real-time image processing applications. Hence, a filter with lower ET is better than a filter having higher ET value when all other performance-measures are identical.

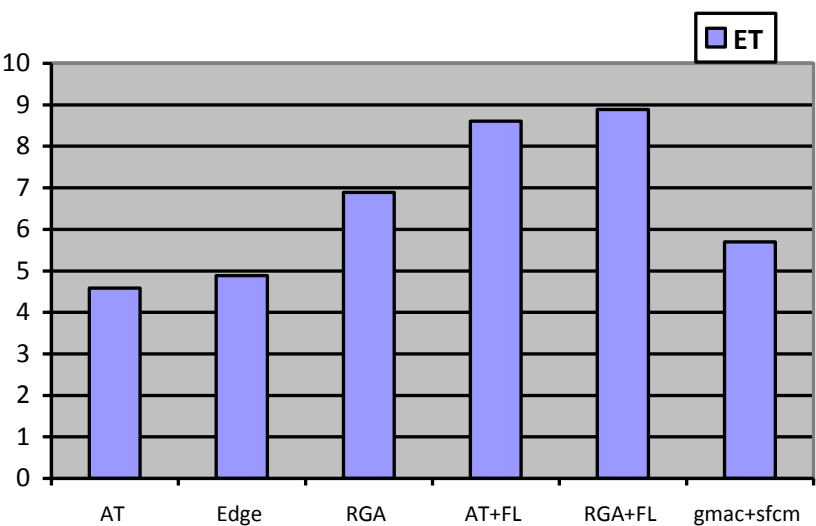

Fig. 6 Elapsed time value for proposed segmentation algorithm

The above figure 6 and table 1 shows the ET value for the different segmentation algorithm. The ET value must low for an better image. Here GMAC+ SFCM gives suitable results. The Figure 7 is shown in annexure page. This gives the image results for different segmentation methods, where the proposed hybrid model gives better and accurate minimization energy level.

\section{CONCLUSION}

In this paper, a hybrid medical image segmentation approach is proposed based on a Global Minimization by active contour and spatial fuzzy clustering model. This approach is composed of two stages. In the first stage, a global minimization of the active contour method is applied, this is based on the unification of image segmentation and image denoising tasks into a global minimization framework. Minimizing this energy provides a unified way to perform image denoising and segmentation .In the second stage, unique FCM algorithm yields better results for segmenting noise free images, but it fails to segment images degraded by noise, outliers and other imaging defects. The optimization property of FCM is improved when it is combined with spatial information (SFCM). It is used to refine segmentation result. The experimental results show that the proposed model can provide close, smooth and accurate final contours with low computational complexity, Energy, Relative \& Discrete Entropy and Mutual Information.

\section{REFERENCES}

[1] D. Jayadevappa, S. Srinivas Kumar, and D. S. Murty, A Hybrid Segmentation Model based on Watershed and Gradient Vector Flow for the Detection of Brain Tumor, International Journal of Signal Processing, Image Processing and Pattern Recognition, Vol. 2, No.3, pp 2942, 2009.

[2] M.C. Clark, L.O. Hall, D.B. Goldgof, MRI Segmentation using Fuzzy Clustering Techniques, IEEE Engineering in Medicine and Biology, Vol. 3,No.5, pp 730-742, 1994.

[3] D. Mumford and J. Shah, Optimal Approximation by Piecewise Smooth Functional and Associated Variational 
Problems, Communications on pure and applied mathematics, Vol. 42, No.5, pp 577-685, 1989.

[4] F.Tonychan, A. Luminitavese, Active Contours Without Edges , IEEE Transaction in Image Processing, Vol.10,No.2, pp 266-277, 2001.

[5] Xavier Bresson, Selim Esedoglu, Pierre Vandergheynst, Jean-Philippe Thiran, and Stanley Osher, Fast Global Minimization of the Active Contour/Snake Model, ACM, Journal of Mathematical Imaging and Vision, Vol. 28, No.2, pp.151-167,2007.

[6] A.Keh-Shih Chuang ,A.Hong-Long Tzeng, A.Sharon Chen, A.Jay Wu, C.Tzong-Jer Chen, Fuzzy c-means clustering with spatial information for image segmentation, Elseiver- Computerized Medical Imaging and Graphics, Vol. 30,No.1, pp 9-15, 2006.

[7] Nassima Mezhoud, Fella Hachouf, "A new hybrid method for medical image segmentation", Journal of Theoritical and Applied Science,Vol.26, No.1, pp 1-15, 2011.

[8] YANG Feng, SUN Xiaohuan, CHEN Guoyue,WEN Tiexiang, An Improved Hybrid Model for Medical Image Segmentation, IEEE conference proceeding, pp 367370, 2008.

[9] Hua Lia, , Anthony Yezzia, A Hybrid Medical Image Segmentation Approach based on Dual-Front Evolution Model , IEEE, conference proceeding, pp 810-813, 2005.

[10] [10] S. Leung and S. Osher, Global Minimization of the Active Contour Model with TV-Inpainting and TwoPhase Denoising, Variational, Geometric, and Level Set Methods in Computer Vision (VLSM), Springer, Vol. 3752 , pp. 149-160, 2005

[11] M. Airouche, L. Bentabet and M. Zelmat, Image Segmentation Using Active Contour Model and Level
Set Method Applied to Detect Oil Spills, Proceedings of the World Congress on Engineering, London, 2009.

[12] L.DzungPham, Spatial Models for Fuzzy Clustering, Computer Vision and Image Understanding, Vol.84, pp.285-297, 2001

[13] Tzong-Jer Chen, Keh-Shih Chuang, Sharon Chen, JengChang Lu, and Ya-Hui Shiao, A Novel Image Smoothing Filter Using Membership Function, Journal of Digital Imaging, Vol.20, No.4,pp 381-392, 2007.

[14] A.Rajendran, R. Dhanasekaran, A Hybrid Method Based on Fuzzy Clustering and Active Contour Using GGVF for Brain TumorSegmentation on MRI Images, European Journal of Scientific Research, Vol.61, No.2 , pp. 305313,2011

[15] Yan Zhang Matuszewski, B.J. Shark, L.-K. Moore, C.J., Medical Image Segmentation Using New Hybrid Level-Set Method, Proceeding of IEEE Conference MEDIVIS '08., pp.71-76,2008.

\section{AUTHORS PROFILE}

Ms. J. Umamaheswari, Research Scholar in Computer Science, Dr. GRD college, Coimbatore. She has 5 years of teaching experience and two years in Research. Her areas of interest include Image Processing, Multimedia and communication. She has more than 3 publications at International level. She is a life member of professional organization IAENG.

Dr. G. Radhamani, Director, Department in Computer Science, Dr. GRD College, Coimbatore. She has more than 5 years of teaching and research experience. She has volume of publications at International level. Her areas of interest include Mobile computing, e-internet and communication. She is a member of IEEE. 


\section{Annexure}

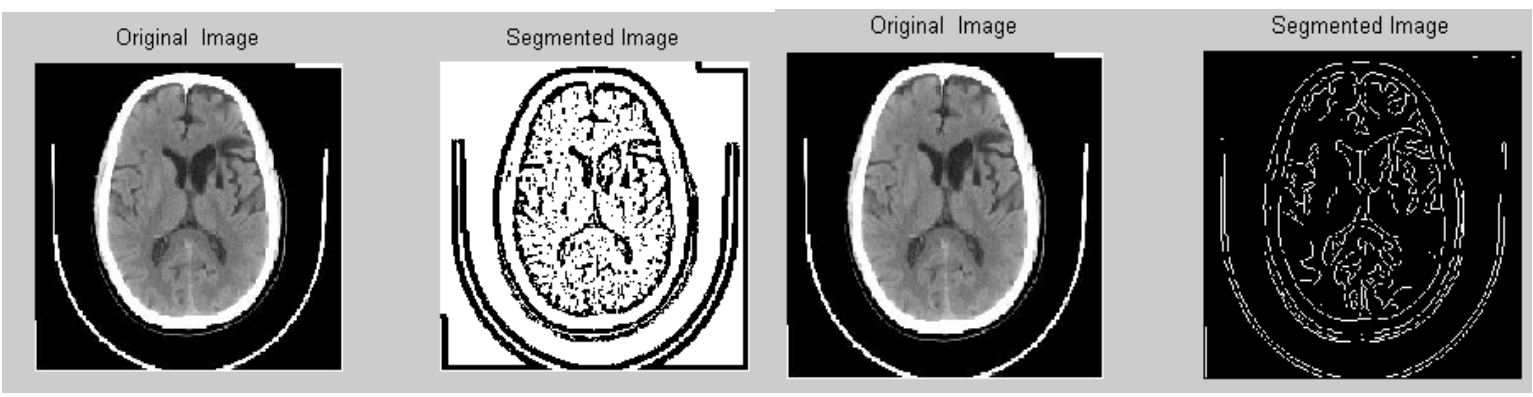

(a)

(b)

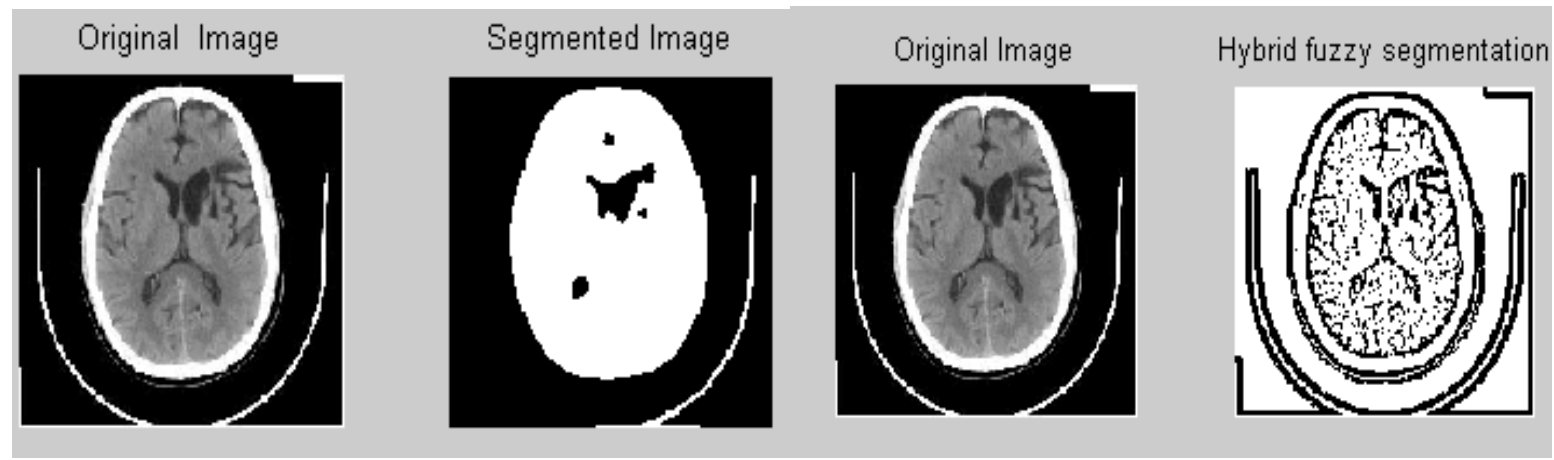

(c)

(d)

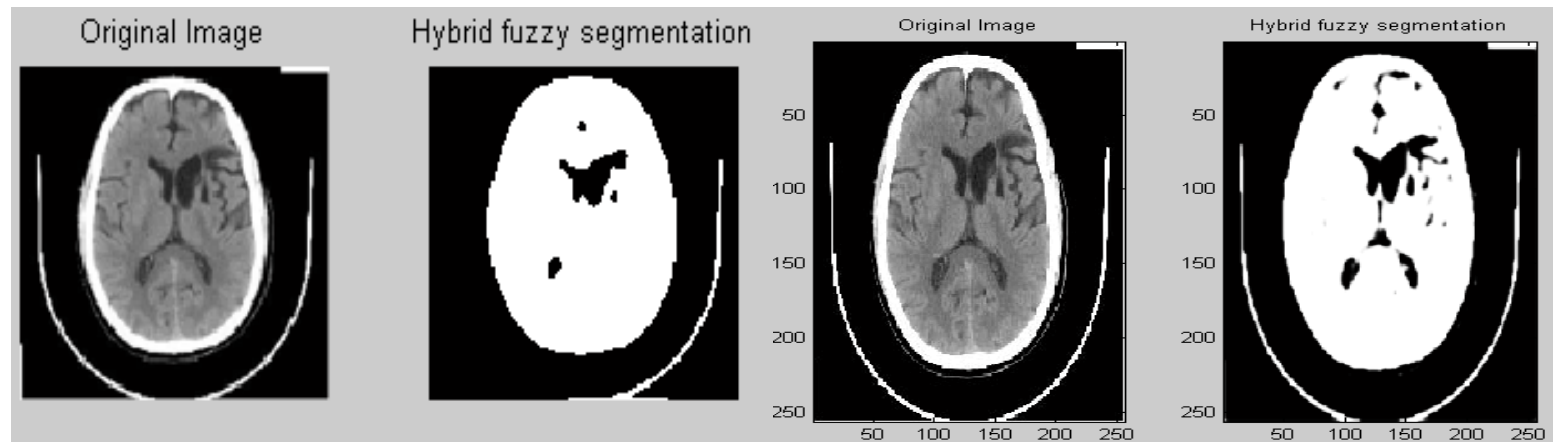

(e)

(f)

Figure 7 Different image segementation algorithm. (a) Adaptive threshold method, (b) Edge Segmentation, (c) Region Growing by Active Contour, (d) Hybrid Segmentation (Adaptive Threshold + FuzzyLevelset), (e) Hybrid Segmentation (Region Growing + FuzzyLevelset), (f) Hybrid Segmentation ( gmac+ sfcm) 